\title{
Improving the static characteristics of hydrodynamic bearings using the viscosity-temperature relations of lubricants
}

\author{
Toshiharu KAZAMA* \\ *College of Design and Manufacturing Technology, Muroran Institute of Technology \\ 27-1 Mizumoto-cho, Muroran, Hokkaido 050-8585, Japan \\ E-mail: kazama@mmm.muroran-it.ac.jp
}

Received 6 December 2015

\begin{abstract}
The possibility of improving the static characteristics of hydrodynamic bearings using the inverse viscosity-temperature relations of liquid lubricants is theoretically examined. A simplified model that comprises the Reynolds equation with a change in the mean oil viscosity across the film as a function of temperature and that is based on the thermal lubrication theory is applied. The solutions are numerically obtained for linear sliding bearings, tapered land bearings, and cosine pad bearings, wherein the temperature distributions are given as known values and the viscosity distributions are determined beforehand. The bearing geometry and temperature profiles are selected as parameters. When the temperature in the converged region close to the minimum clearance is low, the fluid pressure becomes larger; thus, the friction coefficient becomes smaller, whereas the leakage flow rate changes slightly. This behavior may contribute to improving the static characteristics of hydrodynamic bearings.
\end{abstract}

Key words: Tribology, Lubrication, Hydrodynamic bearing, Temperature, Viscosity, Lubricant, Cooling, Control

\section{Introduction}

According to the basics of the lubrication theory, the contributions of the Reynolds equation (Reynolds, 1886) afford three primary mechanisms: the wedge, squeeze, and stretch effects (Soda, 1964; Hamrock, 1994). The positive wedge effect is to produce pressure in the converged region in the sliding direction of the clearance under steady-state conditions; the positive squeeze film effect is to produce pressure when the mating surfaces are approached under unsteady-state conditions, and the stretch effect is to produce pressure under a rather special condition such as rolling processing. Hydrodynamic bearings mainly utilize the wedge effect. According to the lubrication theory, the hydrodynamic pressure is enhanced by higher lubricant viscosity as well as by optimized film shapes. Therefore, to improve the performance of hydrodynamic bearings, the bearing geometry is often highly designed and the sliding surfaces are sometimes optimally textured.

Another way to produce hydrodynamic pressure is through a change in the fluid viscosity and density of the lubricant. The actions based on the viscous wedge (Cameron, 1958) and thermal wedge (Currie et al., 1965) will produce fluid pressures even if the conditions under a parallel film thickness are at the steady state, i.e., $\partial h / \partial x=0$ and $\partial h / \partial \tau=0$, where $h$ is the clearance, $\tau$ is time, and $x$ is the sliding direction. Other approaches are to be generated for the fluid pressure that are caused by cavitation around surface asperities (Dowson, 1957) and slip on solid walls (Spikes, 2003).

In this study, the author theoretically examines the possibility of improving the static characteristics of hydrodynamic thrust pad bearings using inversely the viscosity-temperature relation of liquid lubricants. By means of solving a simplified thermal lubrication model, the effects of temperature distributions and clearance shapes on fluid pressures are investigated. 


\section{Nomenclature}

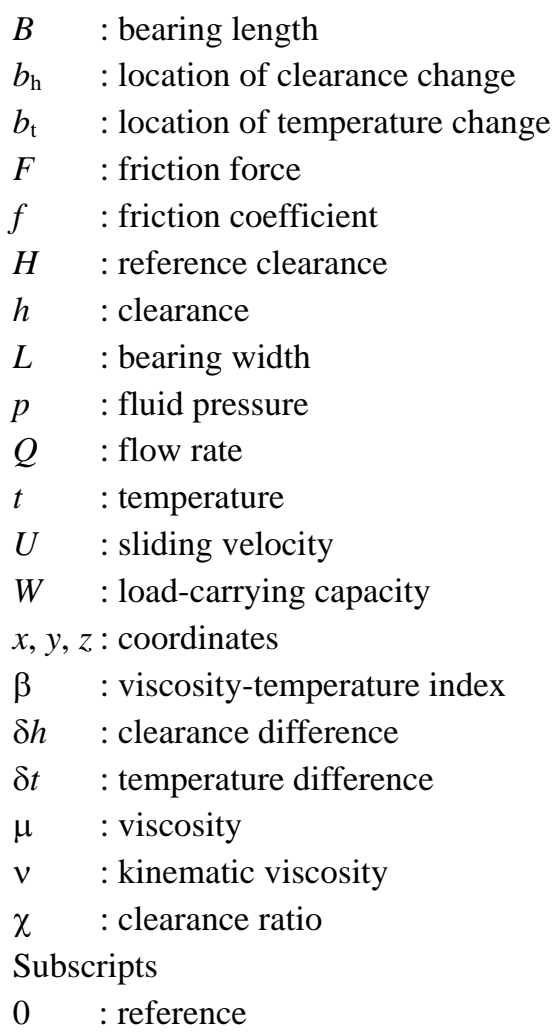

\section{Theory}

\subsection{Basic equations}

Based on the thermohydrodynamic lubrication (THL) theory (Khonsari, 1986), a simplified numerical model is developed to examine the temperature dependence of liquid lubricants on hydrodynamic bearings. The complete THL model (Mitsui et al., 1983) basically comprises the Reynolds, energy, and heat conduction equations, including the changes in physical properties such as viscosity and density as functions of temperature and sometimes pressure (Kazama, 2010). However, if the lubricant temperatures are externally and actively controlled, i.e., when the temperature distributions are given as known values, the energy and heat conduction equations can be omitted from the calculation process. In addition, if the variation in viscosity across the film is ignored, the mean viscosity $\mu_{\mathrm{m}}$ across the film can be defined. Furthermore, if the pressure is not extremely high, it is often assumed that the density, specific heat, thermal conductivity, and thermal expansivity remain constant and that viscosity varies only with temperature.

First, the Reynolds equation applicable to THL problems under steady state is given as follows (Dowson, 1962):

$$
\frac{\partial}{\partial x}\left(G_{2} \frac{\partial p}{\partial x}\right)+\frac{\partial}{\partial y}\left(G_{2} \frac{\partial p}{\partial y}\right)=U \frac{\partial}{\partial x}\left(\frac{G_{3}}{G_{0}}-\int_{0}^{h} \rho d z\right)
$$

where $p$ is the liquid gauge pressure; $U$ is the sliding wall velocity; $\rho$ is the density; and $x, y$, and $z$ are the coordinates in the sliding, perpendicular, and film thickness directions, respectively. When the density $\rho_{0}$ remains constant, and the mean viscosity $\mu_{\mathrm{m}}$ across the film is introduced, the parameters $G_{0}, G_{1}, G_{2}$, and $G_{3}$ are given as follows (Ferron et al., 1983; Kazama et al., 2001): 


$$
\begin{aligned}
& G_{0}=\int_{0}^{h} \frac{d z}{\mu}=\frac{h}{\mu_{m}} \\
& G_{1}=\int_{0}^{h} \frac{z}{\mu} d z=\frac{h^{2}}{2 \mu_{m}} \\
& G_{2}=\int_{0}^{h} \rho \int_{0}^{z} \frac{z}{\mu} d z d z-G_{1} \frac{G_{3}}{G_{0}}=\frac{-\rho_{0} h^{3}}{12 \mu_{m}} \\
& G_{3}=\int_{0}^{h} \rho \int_{0}^{z} \frac{d z}{\mu} d z=\frac{\rho_{0} h^{2}}{2 \mu_{m}}
\end{aligned}
$$

By substituting the simplified expressions of Eq.(2) into Eq.(1), the Reynolds equation is rearranged as

$$
\frac{\partial}{\partial x}\left(\frac{h^{3}}{\mu_{m}} \frac{\partial p}{\partial x}\right)+\frac{\partial}{\partial y}\left(\frac{h^{3}}{\mu_{m}} \frac{\partial p}{\partial y}\right)=6 U \frac{\partial h}{\partial x}
$$

Assuming the change in temperature is limited, the viscosity-temperature relation of lubricating oils can be simply obtained by the exponent function with the index $\beta$ :

$$
\mu=\mu_{0} e^{-\beta\left(t-t_{0}\right)}
$$

where $\mu_{0}$ is the viscosity at temperature $t=t_{0}$.

\subsection{Tapered land and linear sliding bearings}

The clearance $h$ of inclined plane bearings is given by the following expressions with the bearing length $B$ and slope part $b_{\mathrm{h}},(\leq B)$, wherein $b_{\mathrm{h}}<B$ and $b_{\mathrm{h}}=B$ correspond to linear sliding bearings and tapered land bearings, respectively:

$$
\left.\begin{array}{ll}
h=h_{0}+\delta h\left(b_{h}-x\right) / B & ; \quad x<b_{h} \\
h=h_{0} & ; \quad x \geq b_{h}
\end{array}\right\}
$$

Similarly, the temperature distributions $t$ are given a priori by the following expressions:

$$
\left.\begin{array}{lll}
t=t_{0}+\delta t\left(b_{t}-x\right) / B & \text { or } \quad t=t_{0}+\delta t & ; \\
t=t_{0} & & ; \quad x \geq b_{t}
\end{array}\right\}
$$

\subsection{Cosine pad bearings}

The clearance $h$ and temperature $t$ distributions of cosine pad bearings are given by

$$
\begin{aligned}
& h=h_{0}+\delta h \cos (2 \pi x / B) \\
& t=t_{0}+\delta t \sin \left[2 \pi\left(x+b_{t}\right) / B\right]
\end{aligned}
$$

\subsection{Calculation}

The above equations are normalized by the dimensionless values, and the non-dimensional Reynolds equation is given by 


$$
\frac{\partial}{\partial \bar{x}}\left(\frac{\bar{h}^{3}}{\bar{\mu}_{m}} \frac{\partial \bar{p}}{\partial \bar{x}}\right)+\left(\frac{B}{L}\right)^{2} \frac{\partial}{\partial \bar{y}}\left(\frac{\bar{h}^{3}}{\bar{\mu}_{m}} \frac{\partial \bar{p}}{\partial \bar{y}}\right)=\frac{\partial \bar{h}}{\partial \bar{x}}
$$

where $\bar{h}=h / H, \bar{p}=p / S o^{\prime}, \bar{x}=x / B, \bar{y}=y / L$, and $\bar{\mu}_{m}=\mu_{m} / \mu_{0}$.

The side leakage flow rate $\bar{Q}\left[=Q /\left(B^{2} U\right)\right]$, friction force $\bar{F}\left[=F /\left(S o^{\prime} B L\right)\right]$, load-carrying capacity $\bar{W}\left[=W /\left(S^{\prime} B L\right)\right]$, and friction coefficient $f$ are given by the following non-dimensional expressions, respectively:

$$
\begin{aligned}
& \bar{Q}=\frac{H}{L} \int_{0}^{1} \frac{\bar{h}^{3}}{\bar{\mu}}\left(\left.\frac{\partial \bar{p}}{\partial \bar{y}}\right|_{\bar{y}=0}-\left.\frac{\partial \bar{p}}{\partial \bar{y}}\right|_{\bar{y}=1}\right) d \bar{x} \\
& \bar{F}=\frac{H}{B} \int_{0}^{1} \int_{0}^{1}\left(\frac{\bar{h}}{2} \frac{\partial \bar{p}}{\partial \bar{x}}-\frac{1}{6} \frac{\bar{\mu}_{m}}{\bar{h}}\right) d \bar{x} d \bar{y} \\
& \bar{W}=\int_{0}^{1} \int_{0}^{1} \bar{p} d \bar{x} d \bar{y} \\
& f=\frac{\bar{F}}{\bar{W}}
\end{aligned}
$$

Eq.(9) is discretized by the finite difference method, and numerical solutions are obtained by an iterative scheme. The pressure $\bar{p}$ boundary conditions of Eq. (9) are given by $\bar{p}(\bar{x}, 0)=\bar{p}(\bar{x}, 1)=\bar{p}(0, \bar{y})=\bar{p}(1, \bar{y})=0$. For the cavitation conditions, the negative pressure $(\bar{p}<0)$ is instantaneously replaced by zero during the calculation. The mesh size in the $x$ and $y$ directions is $20 \times 20$. Representative numerical parameters are as follows: $H / B=1 \times 10^{-3}, L / B=2, S o^{\prime}=$ $\mu_{0}(U / B)(B / H)^{2}=24 \mathrm{MPa}, t_{0}=40^{\circ} \mathrm{C}$, and $\beta=0.03^{\circ} \mathrm{C}^{-1}$.

\section{Results and Discussion}

\subsection{Tapered land bearings}

Figure 1 illustrates the fluid pressure $\bar{p}$ distributions in terms of the temperature difference $\delta t$ for a linear sliding bearing with the clearance ratio $\chi(=\delta h / H+1)=2$, wherein the temperature changes linearly and the difference $\delta t$ is negative when the inlet temperature is lower than the outlet temperature. The pressure $\bar{p}$ increases with the negative value of $\delta$, and the peak of $\bar{p}$ moves slightly toward the inlet. In general, the temperature of the fluid film is increased by viscous dissipation, which causes a decrease in the viscosity and a decrease in the pressure in the converged region. Therefore, cooling down the film temperature contributes to an increase in the fluid pressure.

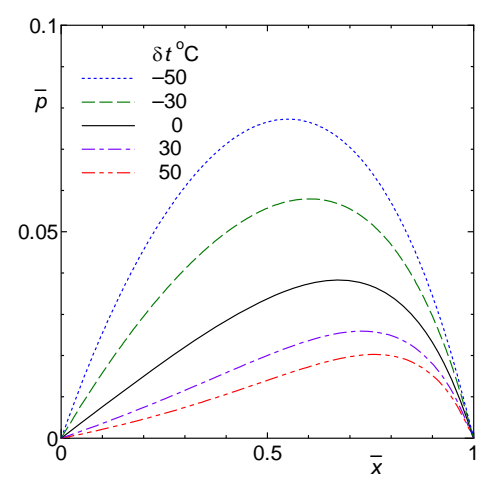

Fig. 1. Effect of temperature difference $\delta$ on pressure distributions $\bar{p}$ for inclined plane bearings $\left(\chi=2, b_{\mathrm{h}} / B=1\right.$, and $\left.b_{\mathrm{t}} / B=1\right)$

Figure 2 illustrates the clearance shape and temperature distributions under the conditions of $\chi=5$ and $\delta t=-30^{\circ} \mathrm{C}$, wherein the locations at which the change in clearance $b_{\mathrm{h}} / B(=0.5)$ and temperature $b_{\mathrm{t}} / B(=0.5)$ coincide. Fig. 3 depicts 
the results of the pressure $\bar{p}$ distributions in terms of the low temperature location $b_{\mathrm{t}} / B$. As $b_{\mathrm{t}} / B$ increases, $\bar{p}$ increases markedly, in particular under the condition of $b_{t} / B \leq 0.5$. This leads to the fluid pressure being effectively increased when the converged region is cooled.

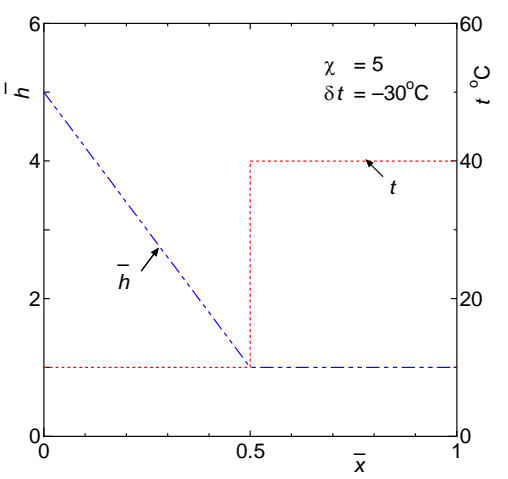

Fig. 2. Clearance shape $\bar{h}$ and temperature distribution $t$ for tapered land bearings $\left(\chi=5, \delta t=-30^{\circ} \mathrm{C}, b_{\mathrm{h}} / B=0.5\right.$, and $\left.b_{\mathrm{t}} / B=0.5\right)$

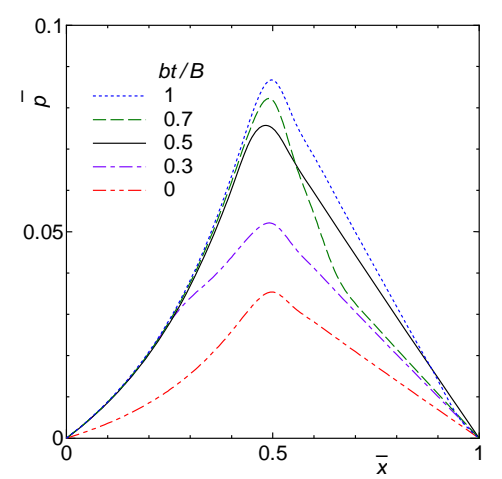

Fig. 3. Effects of low temperature location $b_{t} / B$ on pressure $\bar{p}$ distributions for tapered land bearings $(\chi=5$, $\delta t=$ $-30^{\circ} \mathrm{C}$, and $\left.b_{\mathrm{h}} / B=0.5\right)$

Figures 4 and 5 represent the effects of the low-temperature region $b_{t} / B$ on the friction coefficient $f$ and leakage flow rate $\bar{Q}$ for tapered land bearings, respectively. The coefficient $f$ is, for the higher clearance ratio $\chi$, minimized at about $b_{t} / B=0.5$ as shown in Fig. 4. By contrast, the flow rate $\bar{Q}$ changes less as shown in Fig. 5. One can see that cooling the film in the converged regions contributes the friction coefficient being minimized.

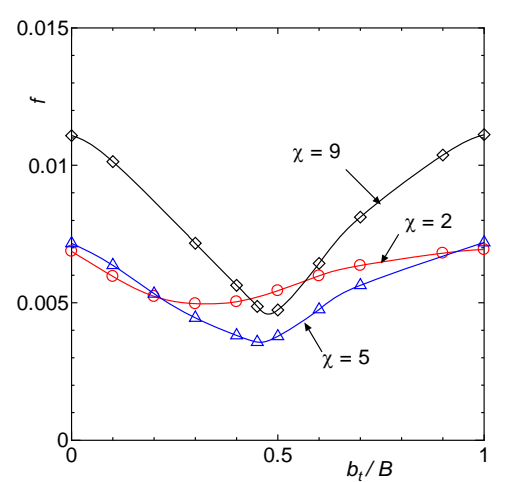

Fig. 4. Effect of low-temperature region $b_{\mathrm{t}} / B$ on friction coefficient $f$ for tapered land bearings $\left(\chi=5, b_{\mathrm{h}} / B=0.5\right.$, $\delta t$ $\left.=-30^{\circ} \mathrm{C}\right)$ 


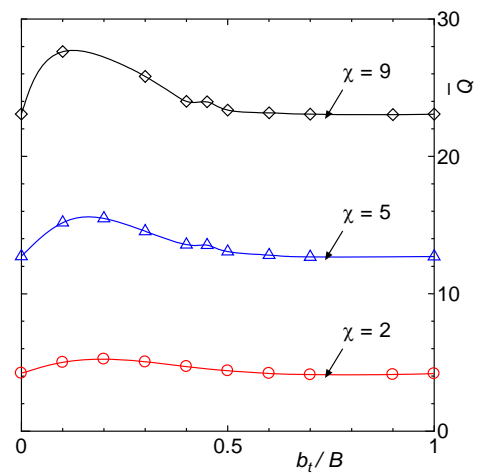

Fig. 5. Effect of low-temperature region $b_{\mathrm{t}} / B$ on leakage flow rate $\bar{Q}$ for tapered land bearings $\left(\chi=5, b_{\mathrm{h}} / B=0.5\right.$, and $\left.\delta t=-30^{\circ} \mathrm{C}\right)$

\subsection{Cosine pad bearings}

Figures 6 and 7 illustrate the pressure $\bar{p}$ distributions for cosine pad bearings. In Fig. 6, as in the distribution for the inclined bearings of Fig. 1, $\bar{p}$ increases as the temperature decreases. In Fig. 7, when the temperature of the converged region is low, $\bar{p}$ is increased.

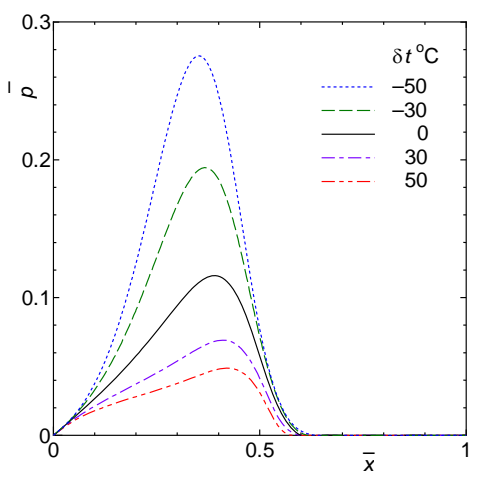

Fig. 6. Effect of temperature difference $\delta t$ on pressure distributions $\bar{p}$ for cosine pad bearings $(\delta h / H=0.5$, $\delta t=$ $-30^{\circ} \mathrm{C}$, and $\left.b_{\mathrm{t}} / \mathrm{B}=0\right)$

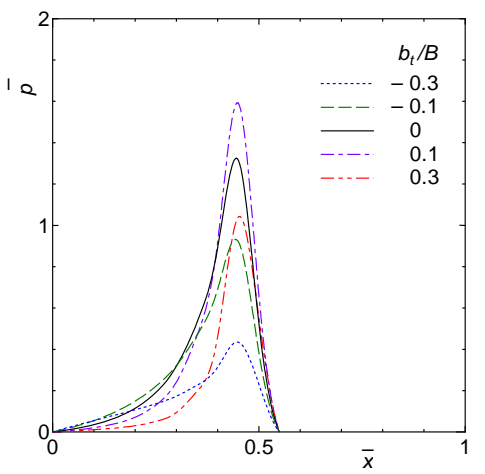

Fig. 7. Effect of low-temperature region $b_{t} / B$ on pressure distributions $\bar{p}$ for cosine pad bearings $(\delta h / H=0.9, \delta t=$ $\left.-30^{\circ} \mathrm{C}\right)$ 
Figure 8 shows the effect of the region $b_{t} / B$ on the friction coefficient $f$ and leakage flow rate $\bar{Q}$ for cosine pad bearings. The coefficient $f$ is minimized at $b_{\mathrm{t}} / B=0.1$, whereas $f$ increases with $\left|b_{\mathrm{t}} / B\right|$. Although the leakage $\bar{Q}$ is minimized at $b_{\mathrm{t}} / B=-0.1$, the change in $\bar{Q}$ is smaller than that in $f$. Note that the present idea based on the inverse usage of the viscosity-temperature relation will enhance the geometrical wedge effect for a specific condition.

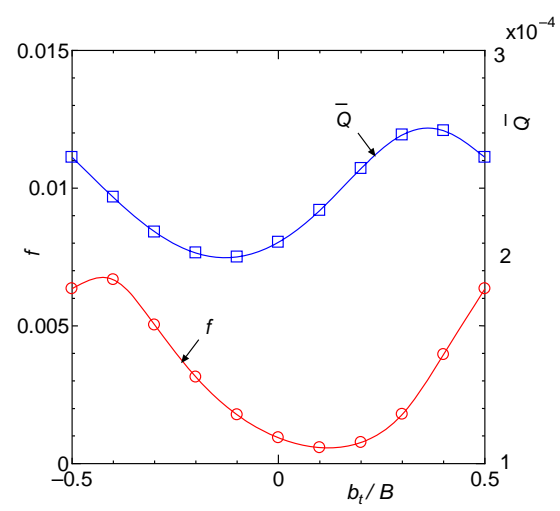

Fig. 8. Effect of low-temperature region $b_{\mathrm{t}} / B$ on friction coefficient $f$ and leakage flow rate $\bar{Q}$ for cosine pad bearings $\left(\delta h / H=0.5, \delta t=-30^{\circ} \mathrm{C}\right.$, and $\left.b_{\mathrm{t}} / B=0\right)$

\section{Conclusions}

With the purpose of improving the performance of hydrodynamic bearings, this study aims to explore the optimum temperature and viscosity distributions using the viscosity-temperature relation of lubricants. Cooling the convergent region in the fluid film may provide an increase in fluid pressures and then a decrease in friction coefficients, which would contribute to the performance of hydrodynamic thrust and journal bearings.

\section{Acknowledgment}

This work was partially supported by JSPS KAKENHI Grant Numbers 22560132 and 25420079.

\section{References}

Cameron, A., The viscosity wedge, ASLE Transactions, Vol. 1 (1958), pp.248-253.

Currie, I. G., Brockley, C. A., and Dvorak, F. A., Thermal wedge lubrication of parallel surface thrust bearings, Transactions of American Society of Mechanical Engineers, Journal of Fluids Engineering, Vol. 87, No. 4 (1965), pp.823-830.

Dowson, D., Investigation of cavitation in lubricating films supporting small loads, Proceedings of the Conference on Lubrication and Wear, Institution of Mechanical Engineers, Göteborg (1957), pp.93-99.

Dowson, D., A generalized Reynolds equation for fluid film lubrication, International Journal of Mechanical Sciences, Vol. 4 (1962), pp.159-170.

Ferron, J., Frene, J., and Boncompain, R., A study of the thermohydrodynamic performance of a plain journal bearing comparison between theory and experiments, Transactions of American Society of Mechanical Engineers, Journal of Lubrication Technology, Vol. 105, No. 3 (1983), pp.422-428.

Hamrock, B. J., Fundamentals of Fluid Film Lubrication, International Editions (1994), McGraw-Hill.

Kazama, T., Thermohydrodynamic lubrication model applicable to a slipper of swashplate type axial piston pumps and motors (Effects of operating conditions), Tribology Online, Vol. 5, No. 5 (2010), pp.250-254.

Kazama, T., Ehret, P., and Taylor, C.M., On the effects of the temperature profile approximation in thermal newtonian solutions of elastohydrodynamic lubrication line contacts, Proceedings of the Institution of Mechanical Engineers, Part J: Journal of Engineering Tribology, Vol. 215, No.1 (2001), pp.109-120. 
Khonsari, M. M., A review of thermal effects in hydrodynamic bearings, Part II: Journal bearings, ASLE Transactions, Vol. 30, No. 1 (1986), pp. 26-33.

Mitsui, J., Hori, Y., and Tanaka, M., Thermohydrodynamic analysis of cooling effect of supply oil in circular journal bearings, Transactions of American Society of Mechanical Engineers, Journal of Tribology, Vol. 105, (1983), pp. 414-421.

Reynolds, O., On the theory of lubrication and its application to Mr. Beauchamp Tower's experiments, including an experimental determination of the viscosity of olive oil, Philosophical Transactions of the Royal Society of London, Vol. 177 (1886), pp.157-234.

Soda, N., Bearings (in Japanese) (1964), Iwanami Shoten, Publishers.

Spikes, H. A., The half-wetted bearing. Part 1: Extended Reynolds equation, Proceedings of the Institution of Mechanical Engineers, Part J: Journal of Engineering Tribology, Vol. 217, No. 1 (2003), pp.1-14. 